\title{
INTERPRETIVE SUMMARIES, AUGUST 2011
}

Some low homogenization pressures improve certain probiotic characteristics of yogurt culture bacteria and Lactobacillus acidophilus LAK. By Muramalla and Aryana, page 3725. Lactobacillus delbrueckii ssp. bulgaricus, Streptococcus salivarius ssp. thermophilus, and Lactobacillus acidophilus are 3 important dairy cultures widely used in preparation of yogurt and other fermented dairy products. Homogenization is a commonly conducted dairy operation. Our attempt was to study how low pressures $(<13.80 \mathrm{MPa})$ affect the acid tolerance, bile tolerance, protease activity, and growth of these dairy cultures for the purpose of pretreating the cultures to enhance targeted probiotic activity. Low homogenization pressure could be used selectively.

Antioxidative effects of whey protein on peroxide-induced cytotoxicity. By Xu et al., page 3739. A concern associated with exercise regards consequences of excess free radical production. The production of reactive oxidative species (ROS) is an outcome of the obligatory increased oxidative metabolism associated with exercise. In addition to established concerns regarding ROS and chronic disease risk, some investigators believe that ROS accumulation delays muscle recovery and may impair performance. Evidence exists that whey protein can help revive athletes after intense exercise and aid recovery, and these areas have become active areas for sports medicine research. We found that whey protein could protect $\mathrm{C}_{2} \mathrm{C}_{12}$ cells from hydrogen peroxide-induced oxidative damage.

Anaerobic digestion of dairy manure influenced by the waste milk from milking operations. $B y$ Wu et al., page 3778. Milk can be digested in an anaerobic digester. Information from the experiments in this study using laboratory-scale anaerobic digesters receiving different levels of milk treatment (from 1 to 19\%) indicated that the added milk can be digested thoroughly and that biogas productivity can be increased by increasing the milk amount in the digester content (from $5.6 \%$ at $1 \%$ milk to $103.8 \%$ at $19 \%$ milk). However, the concentration of $\mathrm{CH}_{4}$ in the biogas produced could decrease slightly with increasing milk content in the digester.

Simultaneous detection of cow and buffalo species in milk from China, India, and Pakistan using multiplex real-time PCR. By Cottenet et al., page 378\%. Asian countries are major producers of cow milk and buffalo milk. To detect cross-contamination for authenticity purposes, a molecular method was developed to simultaneously identify the presence of these two bovine species. Validated on certified references to test its specificity and sensitivity, this method has been applied to 119 Asian milk samples, produced in China, India, and Pakistan.

Angiotensin-converting enzyme inhibitory activity in Mexican Fresco cheese. By Torres-Llanez et al., page 3794. Because diet has a role in the prevention and treatment of hypertension, research has been focused on the manufacture of fermented dairy products with angiotensin-I-converting enzyme inhibitors (ACEI) that may offer antihypertensive activity. Our results supported that Mexican Fresco cheese manufacture with specific lactic acid bacteria strains, such as Enterococcus faecium or a Lactococcus lactis ssp. lactis-Enterococcus faecium mixture, presented significant ACEI activity. Thus, Mexican Fresco cheese presented peptides with potential health benefits.

Reservoir and routes of transmission of Enterobacter sakazakii (Cronobacter spp.) in a milk powder-producing plant. By Jacobs et al., page 3801. Enterobacter sakazakii (Cronobacter spp.) present in powdered infant formula may cause severe or even fatal illness in neonates and infants. However, a reservoir for this organism has not been identified to date. Milk powder is often one of the main ingredients in infant formula. In this study, we showed that textile hose filters (used for powder recovery from exhaust air) in milk powder production can be a reservoir for this organism and can lead to contamination of the final product if the filtered powder is reintroduced into the product.

Analysis of the economically optimal voluntary waiting period for first insemination. By Inchaisri et al., page 3811. The optimal time to start insemination was evaluated by a simulation model using inputs characteristic for Dutch dairy farms. The optimal interval from calving to first insemination of most cows (90\%) was by 10 wk postpartum. However, it was profitable to extend the time to first insemination beyond $10 \mathrm{wk}$ when the cow was in first parity and had a high milk persistency, a delay in peak milk yield time, a delay in the first ovulation time, low cost of milk production, or high insemination cost.

Strategies for time of culling in control of paratuberculosis in dairy herds. By Kudahl et al., page 3824. Paratuberculosis control requires closing transmission routes and culling infectious animals. Many farmers only cull some infectious animals and question the culling time, because premature culling is expensive. Simulations showed that with a $7-$ to $10-y r$ perspective, it was most profitable to delay culling of test-positive cows until milk yield was below $85 \%$ of expected according to parity and lactation stage, es- 
pecially in herds with reproduction problems, but the prevalence was not reduced, only stabilized. With a 10- to 20-yr perspective, prompt culling of test-positive cows was the most profitable strategy and one that also reduced prevalence most effectively.

The relationship between dairy cow hygiene and somatic cell count in milk. By Sant'Anna and Paranhos da Costa, page 3835. This research investigated the hygiene of dairy cows and its relationship with milk quality, providing indicators for the producers to improve management and obtain better economic return. Cleanliness of the leg and udder were associated with reduced somatic cell counts.

Association of standing and lying behavior patterns and incidence of intramammary infection in dairy cows milked with an automatic milking system. By DeVries et al., page 3845. A study was conducted to describe the standing and lying behavior patterns of cows milked with an automatic system and to determine how these patterns relate to the incidence of intramammary infection. Standing and lying behavior patterns of cows were affected by both feed manipulation and their milking activity. Further, postmilking standing time can be managed by providing fresh feed, as well as by pushing up feed, frequently throughout the day. Finally, cows that spend long periods $(>2.5$ h) standing following milking may be at higher risk of acquiring a new intramammary infection.

Effect of solar radiation and flaxseed supplementation on milk production and fatty acid profile of lactating ewes under high ambient temperature. By Caroprese et al., page 3856. Milk yield and milk fatty acid profile were studied after provision of shade and flaxseed supplementation in lactating ewes exposed to high ambient temperature. Only flaxseed supplementation had positive effects on the yield and quality of ewe milk. Moreover, flaxseed supplementation resulted in increased levels of polyunsaturated and functional fatty acids of milk. This finding is of great interest both because consumer demand for dairy products naturally enriched in functional components has grown greatly and because the fatty acid profile of ewe milk undergoes marked deterioration during summer.

Lactoperoxidase activity in milk is correlated with somatic cell count in dairy cows. By Isobe et al., page 3868. Lactoperoxidase (LPO) is a milk protein with antimicrobial properties. The present study was undertaken to examine the correlation between LPO activity and somatic cell count (SCC) in milk in order to use LPO activity as an indicator of mastitis. Higher LPO activity in quarter milk was associated with elevated SCC levels. The percentage of quarter milk with high SCC $\left(4,062 \pm 415 \times 10^{3}\right.$ cells $\left./ \mathrm{mL}\right)$ increased with elevation in LPO activity. These results indicate that the close correlation of LPO activity with SCC in bovine milk may indicate a potential use of the former as an indicator of SCC.

Effects of providing total mixed rations indoors and on pasture on the behavior of lactating dairy cattle and their preference to be indoors or on pasture. By Charlton et al., page 3875. Dairy cows may not be able to meet their nutritional requirements from pasture alone, and may require access to a total mixed ration (TMR). This study offered cows TMR indoors and on pasture. The cows expressed a partial preference of $71 \%$ for pasture, which was not influenced by providing access to the TMR outdoors but did result in an increase in total dry matter intake of the TMR.

Analysis of the influence of variation factors on electrical conductivity of milk in MurcianoGranadina goats. By Diaz et al., page 3885. The development of techniques for early detection of mastitis in the goat farm would allow rapid management of the disease, which would decrease the negative effect on milk quality and safety and on the livestock market economy. The aim was to study the electrical conductivity of gland milk as a tool for detecting mastitis in goats. This study revealed that factors other than health status affect conductivity. We concluded that any detection system based on electrical conductivity of milk should consider these factors (farm, parity, stage of lactation, and interactions), as well as variations specific for each of the animals.

Short communication: Reference values for blood parameters in Holstein dairy cows: Effects of parity, stage of lactation, and season of production. By Cozzi et al., page 3895. Confidence intervals (CI) for blood parameters used for metabolic profile testing were calculated for lactating Holstein cows. Blood samples were collected from 740 cows in 33 herds according to 3 main factors: parity (primiparous vs. multiparous), stage of lactation (early vs. mid), and season of production (summer vs. winter). Specific CI were calculated for blood parameters affected by main factors, whereas general CI were calculated for remaining blood variables. The outcomes of this work should improve the accuracy of the biochemical profile as a tool for dairy practitioners to assess the metabolic status of lactating Holstein cows.

Effect of supplementation with 2-hydroxy4-(methylthio)-butanoic acid isopropyl ester on splanchnic amino acid metabolism and essential amino acid mobilization in postpartum transition Holstein cows. By Dalbach et al., page 3913. The study investigated the effects of feeding a methionine 
analog [2-hydroxy-4-(methylthio)-butanoic acid isopropyl ester] on splanchnic amino acid metabolism and ability of absorbed amino acids to fulfill the need for essential amino acids excreted in milk in very early lactation. Feeding the methionine analog from the day of parturition immediately increased plasma methionine status and increased milk fat content without affecting milk protein yield in the period from parturition to 29 $\mathrm{d}$ in milk. The study showed that a substantial amount of amino acids excreted in milk protein during the postpartum transition period originated from protein mobilization in peripheral tissues (skeletal muscles and uterine involution).

Contribution of exopeptidases to formation of nonprotein nitrogen during ensiling of alfalfa. $B y$ Tao et al., page 3928. The experiment was conducted to investigate the exopeptidase classes in alfalfa (Medicago sativa L.) leaves, and to determine their contribution to the formation of nonprotein nitrogen components during ensiling. Results of the current study indicated that 5 classes of exopeptidase exist in alfalfa, and dipeptidase, carboxypeptidase, and tripeptidyl-peptidase are the principal enzymes hydrolyzing the proteins of the ensiled forage. These findings might provide useful information for developing proteolysis inhibiting additives for ensiled forage in commercial settings.

Fatty acid intake alters growth and immunity in milk-fed calves. By Hill et al., page 3936. This study provides further evidence that the supplementation of milk replacers with NeoTec4, a blend of butyrate, coconut, and flax oils, improves growth rate and feed efficiency and reduces scours and treatments for clostridium sickness in young calves. NeoTec4 altered some immune and inflammatory responses of young calves following vaccination, which may affect their ability to handle a disease challenge and might explain why scours are reduced. This immune response occurred in as little as $6 \mathrm{~d}$ of feeding NeoTec4. Also, when NeoTec4 was fed via dry starter and to weaned calves, growth rate and feed efficiency were improved.

Grazing management and supplementation effects on forage and dairy cow performance on cool-season pastures in the southeastern United States. By Macoon et al., page 3949. This study evaluated grazing of lactating Holstein cows on pastures of mixed rye and annual ryegrass compared with those grasses mixed with red and crimson clover. Stocking rate and supplement feeding rates were also tested. We found that milk production was not improved by adding clovers to mixed cool-season grass pastures and that stocking rate had the greatest effect on milk production. Low stocking rate resulted in greater production per animal but high stocking rate had greater production per land area. However, supplementation with concentrates was needed to ensure maintenance of cow weight and body condition.

Effects of the percentage of concentrate on rumen fermentation, nutrient digestibility, plasma metabolites, and milk composition in mid-lactation goats. By Serment et al., page 3960. The concentrate proportion of dairy goat rations has increased in recent years and may have adverse effects on rumen fermentation and milk quality. The aim of this work was to study the effect of high-concentrate diets on intake pattern, rumen fermentation, digestion, and milk quality for $10 \mathrm{wk}$. Compared with a low-concentrate diet, milk and protein yields increased with the high-concentrate diet, whereas milk fat content, rumen $\mathrm{pH}$, and ammonia concentration decreased. Such a study is essential to provide data to develop empirical or mechanistic models aimed at predicting responses to dietary change at the digestive, metabolic, and mammary levels.

Effect of applying bacterial inoculants containing different types of bacteria to corn silage on the performance of dairy cattle. By Arriola et al., page 3973. This study examined the effect of applying different bacterial inoculants at the time of ensiling on the quality and aerobic stability of corn silage and performance of lactating dairy cows. Application of Lactobacillus buchneri 40788 improved the aerobic stability of the corn silage but none of the inoculant treatments affected feed intake, milk yield, or efficiency of feed utilization by dairy cows.

Effects of ruminal ammonia and butyrate concentrations on reticuloruminal epithelial blood flow and volatile fatty acid absorption kinetics under washed reticulorumen conditions in lactating dairy cows. By Storm et al., page 3980. The effect of changes in reticuloruminal epithelial blood flow, induced by ruminal $\mathrm{NH}_{3}$ and butyrate, on the absorption of volatile fatty acids (VFA) was studied under washed rumen conditions in lactating dairy cows. High amounts of butyrate in the rumen increased epithelial blood flow and ruminal disappearance of propionate together with the net portal appearance of propionate and isobutyrate. Rumen epithelial blood flow is important in control of VFA absorption from the rumen, and the present study points to epithelial blood flow as an important variable for modeling ruminal VFA kinetics.

Dietary molasses increases ruminal pH and enhances ruminal biohydrogenation during milk fat depression. By Martel et al., page 3995. Highconcentrate diets were fed to lactating Holstein cows to induce milk fat depression (MFD), and corn grain was replaced with varying levels of molasses to study the potential to alleviate MFD. Cows consuming di- 
ets containing molasses at $5 \%$ of dry matter showed increased milk fat concentration compared with the control high-concentrate diet. Molasses increased ruminal $\mathrm{pH}$, decreased total ruminal volatile fatty acid concentration, decreased the yield of milk trans-10 C18:1, and increased the yield of milk trans-11 C18:1. Results indicated that molasses may partially alleviate MFD induced by high-concentrate diets by altering ruminal $\mathrm{pH}$ and pathways of ruminal biohydrogenation.

Effectiveness of extruded rapeseed associated with an alfalfa protein concentrate in enhancing the bovine milk fatty acid composition. By Dang Van et al., page 4005. This experiment demonstrates that extruded rapeseed associated with an 18:3 n-3-rich alfalfa protein concentrate can be a valuable alternative to extruded linseed, as an unsaturated fatty acid source aimed at improving the bovine milk fat profile in general, and its 18:3 n-3 content in particular. It also shows that the transfer efficiency of 18:3 n-3 from diet to milk is higher for alfalfa protein concentrate compared with extruded linseed. The present study thus indicates that at least 2 economically viable feeding alternatives exist to improve the nutritional profile of milk fat.

Effect of rumen-protected choline on performance, blood metabolites, and hepatic triacylglycerols of periparturient dairy cattle. By Zom et al., page 4016. The start of lactation coincides with an increased concentration of triacylglycerols in the liver, which has been associated with other health and reproduction problems in dairy cattle. An experiment was carried out to test the hypothesis that an increased supply of choline through feeding rumen-protected choline reduces the accumulation of triacylglycerols after calving, which may improve animal performance. Choline supplementation reduced the concentration of triacylglycerols in liver after calving. Dairy cows supplemented with rumen-protected choline had higher dry matter intake and milk protein production at the start of lactation than unsupplemented dairy cows.

Persistency of methane mitigation by dietary nitrate supplementation in dairy cows. By van Zijderveld et al., page 4028. Methane is a powerful greenhouse gas, and enteric methane production in dairy cows represents both an environmental burden and a loss of dietary energy to the dairy cow. In this paper, the effectiveness of dietary nitrate in methane mitigation in lactating dairy cows is described. Methane production was persistently lowered $(-16 \%)$ over an 89-d period by dietary nitrate inclusion, but this did not result in increased availability of dietary energy to the animal.

Effect of parity and stage of lactation on feed sorting behavior of lactating dairy cows. $B y$
DeVries et al., page 4039. The objectives of this study were to determine if feed sorting differs between primiparous and multiparous cows when individually housed and fed, if sorting changes from the period of peak lactation to peak dry matter intake, and whether this feed sorting affects efficiency of production. The results demonstrate that feed sorting remained consistent in all cows across days in milk and that this sorting behavior may affect the efficiency of milk production as well as milk fat percentage. Further, primiparous cows engage in more sorting against the longest ration particles and for the smallest ration particles compared with multiparous cows during this period.

Effects of supplementation with yeast culture and enzymatically hydrolyzed yeast on performance of early lactation dairy cattle. By Nocek et al., page 4046. Yeast culture and yeast culture with enzymatically hydrolyzed yeast were fed to evaluate production performance and health in dairy cattle. Cows in both yeast treatments yielded more milk than nonsupplemented cows. Milk protein percentage and yields were elevated for enzymatically hydrolyzed yeast compared with the control. Somatic cell count was decreased with enzymatically hydrolyzed yeast during wk 8 to 14 postpartum. Supplementation of early lactation cows with yeast culture improved production performance; however, further performance and mammary gland health benefits were realized when cows were additionally supplemented with enzymatically hydrolyzed yeast.

Effect of replacing solvent-extracted canola meal with high-oil traditional canola, higholeic acid canola, or high-erucic acid rapeseed meals on rumen fermentation, digestibility, milk production, and milk fatty acid composition in lactating dairy cows. By Hristov et al., page $405 \%$. This study investigated the effect of inclusion of highoil canola or rapeseed meals with a variable fatty acid profile in the diet of lactating dairy cows on rumen fermentation, animal performance, and milk fatty acid composition. High-oil canola or rapeseed meals, which are likely to come from small-scale biodiesel plants where oil is cold pressed without hexane extraction, fed at levels at or above 12 to $13 \%$ of dietary dry matter may decrease feed intake and milk production, but can be used to alter milk fatty acid composition in lactating dairy cows.

Short communication: Effects of supplementation with pomegranate seed pulp on concentrations of conjugated linoleic acid and punicic acid in goat milk. By Modaresi et al., page 4075. Nutritional quality of milk can be assessed based on its fatty acid profile and protein content. One of the desirable attributes of some fatty acids such as conju- 
gated linoleic acid is their anticarcinogenic properties. An experiment was conducted to investigate the fatty acid profile in goat milk supplemented with various levels of pomegranate seed pulp. Supplementation with pomegranate seed pulp increased concentrations of conjugated linoleic acid and punicic acid, which have beneficial health effects in humans.

Bimodality and the genetics of milk flow traits in the Italian Holstein-Friesian breed. By Samoré et al., page 4081. The pattern of milk flow in dairy cows holds great interest for genetic selection. This is supported by the consistent heritability of traits controlling milk flow and their genetic correlation with production, udder type traits, and somatic cell score (an udder health indicator). Based on the genetic associations found in this study, intermediate milk flow rates may be optimal. Genetic parameters support the possible use of bimodality, defined as the delayed milk ejection at the start of milking, for genetic selection.

Effect of type traits on functional longevity of Czech Holstein cows estimated from a Cox proportional hazards model. By Zavadilová et al., page 4090. The objective of this study was to evaluate the relationship between type traits and functional longevity in Czech Holstein cows using a Cox proportional hazard model. Twenty linear traits, 6 composite type traits, and height at sacrum were analyzed. Among the composite traits, dairy form, udder, and final score had the strongest relationships with longevity. Among the linear traits, body condition score, udder depth, and angularity had the most important relationships with longevity, whereas foot angle and bone quality showed negligible effects.

Ex situ conservation of Holstein-Friesian cattle: Comparing the Dutch, French, and US germplasm collections. By Danchin-Burge et al., page 4100. The contraction in Holstein-Friesian genetic diversity has been documented and mitigation initiated. One element of mitigation has been the establishment of cryopreserved collections of germplasm for this breed in national gene banks. To better understand the diversity captured in gene banks, genetic variability of Holstein-Friesian collections within and among countries was assessed and compared using active bulls in each country. A substantial part of the US, French, and Dutch collections were genetically similar. However, the US collection represented an interesting reservoir of Holstein-Friesian genetics from the past, illustrating how gene banks can support livestock genetic diversity conservation by cryopreserving founders of breeds or breeding populations.
Use of female information in dairy cattle genomic breeding programs. By Mc Hugh et al., page 4109. Genomic selection is now the method of choice for many national dairy genetic evaluation systems. However, few studies have attempted to quantify the optimal breeding program to fully exploit this new approach. The aim of this study was to quantify the effect of alternative breeding schemes on genetic gain and inbreeding, with particular emphasis on the contribution of females to the breeding scheme. Results from this study clearly show the benefit of genomic selection in increasing genetic gain, which is further augmented by the inclusion of female information.

A genome scan for quantitative trait loci affecting body conformation traits in Spanish Churra dairy sheep. By Gutiérrez-Gil et al., page 4119. A genome scan was performed to detect quantitative trait loci (QTL) influencing body conformation traits in Spanish Churra sheep. Following a daughter design, 739 ewes belonging to 11 half-sib families were scored for 5 linear traits related to body conformation: stature, rear legs-rear view, foot angle, rump width, and general appearance. The analysis of 181 microsatellites allowed for the identification of 7 putative QTL. These regions can be used as milestones for future studies aimed toward the identification of markers associated with these functional traits.

Genetic analyses of protein yield in dairy cows applying random regression models with time-dependent and temperature $\times$ humiditydependent covariates. By Brügemann et al., page 4129. Because of the effect of global warming, heat stress in dairy cows is a topic of increasing concern. The aim of this study was to assess the impact of heat stress in terms of temperature and relative humidity on the estimates of genetic parameters for daily protein yield of German dairy cows. Genetic correlations for protein yield across heat stress levels were close to 1 . This result disproved any concerns related to genotype $x$ environment interaction. A clear trend was observed that an increase of heat stress was associated with a decrease in heritabilities and additive-genetic variances.

Optimal strategies for the use of genomic selection in dairy cattle breeding programs. $B y$ Wensch-Dorendorf et al., page 4140. Genomic selection is a new tool within breeding programs for dairy cattle. Until now, costs have prevented breeding organizations from relying on a fully genotyped population, and careful planning should assess the competitiveness of different strategies for genotyping of potential candidates for selection. A stochastic simulation revealed that the 
focus of genotyping should be on genotyping of male calves to identify future artificial insemination bulls. Preselection steps should be used to narrow the pool of young males to be genotyped.

Phenotypic and genetic variability of production traits and milk fatty acid contents across days in milk for Walloon Holstein first-parity cows. By Bastin et al., page 4152. The milk fatty acid profile of dairy cows and its sources of variation present multiple interests for the improvement of economic, nutritional, technological, and sensory quality of milk fat but also for better understanding of the metabolism and the environment of cows. Therefore, the objective of this study was to assess the phenotypic and genetic variability of milk fatty acid contents throughout the lactation. Results indicated that the fatty acid profile in milk changed during lactation phenotypically and genetically, and emphasized the relationship between the physiological status of cow and milk composition.

Marker-assisted breeding value estimation for mastitis resistance in Finnish Ayrshire cattle. By Mulder et al., page 4164. In this study we demonstrate marker-assisted breeding value estimation combining data of genotyped and ungenotyped animals for mastitis resistance in Finnish Ayrshire cattle. Five quantitative trait loci regions were modeled using identical-by-state (IBS) or identical-by-descent (IBD) haplotypes. Using IBS haplotypes yielded better accuracy of estimated breeding values than using IBD haplotypes, but were similar to those using conventional breeding values. In this study, conventional breeding value estimation is advocated, but in other cases, the use of IBS haplotypes may increase accuracy of estimated breeding values and is feasible in large populations with genotyped and ungenotyped animals.

Differential expression of heat shock protein genes and their splice variants in bovine preimplantation embryos. By Zhang et al., page 4174 . The objective of this study was to profile the expression of 17 heat-shock protein genes in bovine embryos that differ in morphology and developmental status, and to carry out an association analysis with fertility traits. All 17 genes showed differential expression between the embryo groups, and 2 genes were associated with fertilization and blastocyst rates.

Prediction of $\beta$-lactoglobulin genotypes based on milk Fourier transform infrared spectra. $B y$ Rutten et al., page 4183. $\beta$-Lactoglobulin genotypes are associated with milk composition; for example, the relative casein content. In this study we investigated whether $\beta$-lactoglobulin genotypes can be predicted based on routinely recorded milk Fourier transform infrared spectra. The results show that $\beta$-lactoglobulin genotypes can be predicted correctly for, on average, $74 \%$ of the cows. Therefore, the presented methodology provides an inexpensive method to predict $\beta$-lactoglobulin genotypes on the individual cow level and might prove valuable in on-farm selection decisions related to milk composition.

Genomic and pedigree-based genetic parameters for scarcely recorded traits when some animals are genotyped. By Veerkamp et al., page 4189. Estimates of heritability and genetic variances were lower when based on genomic relationships compared with pedigree relationships, but genetic parameters were more accurate. Including both genotyped and nongenotyped animals by merging genomic and pedigree relationships yielded the most accurate estimates. Genotyping animals was preferable to recording extra records in terms of accuracy of estimated genetic parameters.

Multiple-trait genomic evaluation of linear type traits using genomic and phenotypic data in US Holsteins. By Tsuruta et al., page 4198. Genomic breeding values for 18 linear type traits were estimated with multiple-trait single-step procedures using genotypes for US Holstein bulls and phenotypes and pedigree information for US Holstein cows. Genomic evaluations with multiple-trait models were more accurate than those with single-trait models. Modifying a weight for pedigree relationships of genotyped animals reduced bias in genomic evaluations.

Genetic parameters of coagulation properties, milk yield, quality, and acidity estimated using coagulating and noncoagulating milk information in Brown Swiss and Holstein-Friesian cows. By Cecchinato et al., page 4205. Milk coagulation properties (MCP) are commonly defined as milk coagulation time and curd firmness. A critical feature of MCP data is the presence of noncoagulating (NC) milk records. No previous study investigated genetic relationships between $\mathrm{MCP}$ and other milk traits (i.e., milk yield and composition, somatic cell score, and acidity) including NC milk information in the statistical analysis. Genetic correlations for MCP with milk yield and milk composition traits were low or moderate. Genetic correlations of MCP with somatic cell score were large, and even larger were those with $\mathrm{pH}$ and titratable acidity.

Short communication: Expression and alternative splicing of POU1F1 pathway genes in preimplantation bovine embryos. By Laporta et al., page 4220. Proper development of preimplantation embryos is critical to the success of conception in cattle. In this study, expression levels of genes from the POU1F1 pathway were estimated, and OPN and STAT5A were 
found to be differentially expressed between blastocysts and degenerate embryos, which differ in their morphology and developmental status.

Short communication: Refinement of genetic regions associated with Mycobacterium avium subspecies paratuberculosis tissue infection and tolerance to Johne's disease. By Zanella et al., page 4230. Mycobacterium avium ssp. paratuberculosis is the causative agent of Johne's disease (bovine paratuberculosis) and accounts for annual losses of over $\$ 200$ million in the dairy industry. This study investigated chromosomal regions associated with tolerance and tissue infection with $M$. avium ssp. paratuberculosis in Holstein cows. The identification of genetic markers associated with infection and tolerance to bovine paratuberculosis could be used to select animals to reduce animal morbidity and mortality, thereby reducing economic losses caused by this disease.
Comparison of bulk-tank standard plate count and somatic cell count for Wisconsin dairy farms in three size categories. By Ingham et al., page $423 \%$. In order to compare the quality of milk from different size categories of Wisconsin dairy farms, we analyzed the monthly reported bulk-tank standard plate count (SPC) and somatic cell count (SCC) results from Wisconsin dairy farms from February to December 2008. Farms were grouped into 3 size categories based on available size-tracking information: small $=$ no more than 118 cows; large $=119$ to 713 cows; and confined animal feeding operation (CAFO; at least 714 cows). On average, the lowest median, mean, 90th percentile, and maximum SPC and SCC were found on CAFO farms, followed by large, and then small farms. Using the quality and farm-size criteria employed in this study, the claim that smaller Wisconsin farms produce higher quality milk than larger Wisconsin farms cannot be supported. 